\title{
Unilateral Choanal Atresia: Indications of Long-Term Olfactory Deficits and Volumetric Brain Changes Postsurgically
}

\author{
Charalampos Georgiopoulos ${ }^{\mathrm{a}, \mathrm{b}}$ Martina Postler ${ }^{\mathrm{a}}$ Philippe Rombaux ${ }^{\mathrm{c}}$ \\ Volker Gudziol $^{\mathrm{a}}$ Nasreddin Abolmaali ${ }^{d}$ Thomas Hummel ${ }^{\mathrm{a}}$ \\ aSmell and Taste Clinic, Department of Otorhinolaryngology, TU Dresden, Dresden, Germany; ${ }^{b}$ Department of \\ Radiology and Department of Medical and Health Sciences, Linköping University, Linköping, Sweden; 'Department \\ of Otorhinolaryngology, Cliniques Universitaires Saint Luc, Brussels, Belgium; IInstitute for Diagnostic and \\ Interventional Radiology and Nuclear Medicine, St. Josef Hospital Bochum, Ruhr University Bochum, Bochum, \\ Germany
}

\section{Keywords}

Choanal atresia · Olfaction · Voxel-based morphometry

\begin{abstract}
Background: Very few studies have investigated whether unilateral choanal atresia is associated with permanent olfactory deficits. Objective: This study aimed to evaluate the olfactory performance of patients with unilateral choanal atresia postsurgically. Methods: Three patients with unilateral atresia were examined in terms of olfactory performance with the Sniffin' Sticks test (odor identification, threshold, and discrimination), size of the olfactory bulb, and volumetric brain changes. Results: All patients demonstrated significantly lower olfactory performance in terms of odor threshold on the same side with the choanal atresia. Grey matter reductions were found ipsilaterally in the hippocampus. Conclusions: This pilot study indicates that persistent olfactory deficits and volumetric brain changes are present in patients with unilateral choanal atresia.
\end{abstract}

(c) 2021 The Author(s)

Published by S. Karger AG, Basel

karger@karger.com www.karger.com/orl

Karger!"

GOPEN ACCESS
C) 2021 The Author(s).

Published by S. Karger AG, Basel

This is an Open Access article licensed under the Creative Commons Attribution-NonCommercial-4.0 International License (CC BY-NC) (http://www.karger.com/Services/OpenAccessLicense), applicable to the online version of the article only. Usage and distribution for commercial purposes requires written permission.

\section{Introduction}

Congenital choanal atresia is an uncommon craniofacial abnormality, with a prevalence of approximately $0.82-0.91$ per 10,000 live births $[1,2]$. It is characterized by mixed bony and membranous obstructions, causing the narrowing and closure of the posterior choanae of the nose [3]. This malformation can occur unilaterally or bilaterally, with the latter being relatively less common [4]. Moreover, it is known to co-occur with a number of other disorders, the most common of which is CHARGE (coloboma, heart defects, atresia choanae, retarded growth, genitourinary abnormalities, and ear anomalies/ deafness) syndrome [5].

Bilateral choanal atresia, being characterized by asphyxia, stridor, and paradoxical cyanosis, is a life-threatening condition that is generally diagnosed at birth. On the contrary, unilateral atresia is usually diagnosed during the first 2 years of life and is characterized by persistent nasal discharge [3]. Rare cases of choanal atresia diagnosed in adulthood have also been reported [6]. Diagnosis of choanal atresia is established after clinical examination, nasal endoscopy, and CT of the paranasal

Correspondence to:

Charalampos Georgiopoulos, charalampos.georgiopoulos@liu.se 
sinuses and the skull base. Treatment includes a variety of surgical procedures, and there is to date no standard surgical protocol for these patients $[3,4]$.

Choanal atresia has significant effects on olfactory function because it prevents odors from reaching the olfactory cleft. Based on the plasticity of the olfactory system, it can be assumed that atresia modulates the development of the olfactory system $[7,8]$. Yet, few studies have evaluated the olfactory performance of patients with choanal atresia postsurgically. Two studies on children have demonstrated impaired olfaction (either hyposmia or anosmia) and abnormalities in the olfactory bulbs, which were either absent or hypoplastic $[9,10]$. Adults with bilateral atresia tend to demonstrate permanent olfactory deficits, whereas those with unilateral atresia are reported to have normal olfaction [11, 12]. Nevertheless, it is important to highlight that the aforementioned studies in adult population include a very limited number of participants. Moreover, to the best of our knowledge, there are to date no studies that have evaluated potential morphological brain alterations in patients with choanal atresia. Here, we present the results regarding olfactory function and brain volumetry from 3 patients with unilateral choanal atresia.

\section{Materials and Methods}

Three participants were included in this pilot study. Patient 1 , a 50-year-old woman, was surgically treated at the age of 39 for left-sided choanal atresia. Patient 2, a 25-year-old man, was surgically treated at the age of 22 for right-sided choanal atresia. Patient 3 , a 20 -year-old man, was surgically treated at the age of 9 for rightsided choanal atresia. All patients underwent olfactory evaluation and MR) examination 10 (patient 1), 2 (patient 2), and 9 (patient 3 ) years postsurgically. None of the patients had other congenital anomalies that are associated with choanal atresia, such as CHARGE syndrome, Apert's syndrome, DiGeorge syndrome, trisomy 18, Treacher Collins syndrome, or camptomelic dysplasia [13].

Upon questioning, none of the participants reported major troubles with olfaction. Olfactory evaluation was performed with the Sniffin' Sticks testing battery (Burghart Messtechnik GmbH, Wedel, Germany), involving subtests for odor threshold (T), discrimination (D), and identification (I) [14]. Each nostril was tested separately, yielding a TDI (threshold, discrimination, and identification) sum score for each side. Nostrils were tested in a random order.

MRI was performed using a GE Discovery MR 750, 3-Tesla scanner, with an 8-channel head coil, including high-resolution 3D T1-weighted images (repetition time: $7.9 \mathrm{~ms}$, echo time: $3 \mathrm{~ms}$, slice thickness: $1 \mathrm{~mm}$, and voxel size: $1 \mathrm{~mm}$ isotropic) and coronal T2-weighted images (repetition time: 4,000 ms, echo time: 123 $\mathrm{ms}$, and slice thickness: $2 \mathrm{~mm}$ ). T1-weighted images were analyzed with voxel-based morphometry (VBM), by employing the Com-
Table 1. Olfactory performance and volumetric results

Patient 1 Patient 2 Patient 3

\begin{tabular}{llll}
\hline Healthy side & & & \\
$\quad$ TDI score & 30.25 & 30 & 33.25 \\
Threshold score & 8.25 & 6 & 7.25 \\
Discrimination score & 10 & 11 & 12 \\
$\quad$ Identification score & 12 & 13 & 14 \\
$\quad$ Size of olfactory bulb, $\mathrm{mm}^{3}$ & 43.42 & 43.66 & 47.66 \\
$\quad$ Size of entorhinal cortex, $\mathrm{cm}^{3}$ & 1.96 & 2.24 & 2.67 \\
$\quad$ Size of hippocampus, $\mathrm{cm}^{3}$ & 3.01 & 3.24 & 3.77 \\
$\quad$ Size of amygdala, $\mathrm{cm}^{3}$ & 0.67 & 0.79 & 0.99 \\
Side of choanal atresia & & & \\
$\quad$ TDI score & 20.75 & 19.25 & 27.75 \\
$\quad$ Threshold score & 2.75 & 1.25 & 3.75 \\
$\quad$ Discrimination score & 8 & 6 & 14 \\
$\quad$ Identification score & 10 & 12 & 10 \\
$\quad$ Size of olfactory bulb, $\mathrm{mm}^{3}$ & 33.88 & 41.93 & 44.33 \\
$\quad$ Size of entorhinal cortex, $\mathrm{cm}^{3}$ & 2.09 & 1.97 & 2.43 \\
$\quad$ Size of hippocampus, $\mathrm{cm}^{3}$ & 2.75 & 2.94 & 3.34 \\
Size of amygdala, $\mathrm{cm}^{3}$ & 0.76 & 0.79 & 0.94 \\
\hline
\end{tabular}

putational Anatomy Toolbox (CAT12; http://www.neuro.uni-jena.de/cat), a free extension to Statistical Parametrical Mapping 12 (SPM12; https://www.fil.ion.ucl.ac.uk/spm/). VBM was performed with the default settings, as described by the developer of CAT12. VBM data were analyzed with a region of interest (ROI) approach, by extracting the mean grey matter volume within predefined olfactory brain regions. The following ROIs were employed, as defined by the Neuromorphometrics, Inc. atlas (http:// Neuromorphometrics.com/), part of CAT12: entorhinal cortex, hippocampus, and amygdala. Coronal T2-weighted images were used to calculate the volume of the olfactory bulb, as previously described [15].

Statistical analysis regarding the TDI-score, the volume of the olfactory bulb, and the mean grey matter volume within the aforementioned ROIs was performed with the paired $t$ test (the healthy side was compared to the side of choanal atresia). Statistical analysis of the behavioral and the volumetric data was performed with IBM $^{\circledR}$ SPSS Statistics version 27 , and statistical significance was set at $p<0.05$ with 1,000 bootstrap samples.

\section{Results}

The patients had significantly lower olfactory performance in terms of odor threshold ( $p=0.001$, based on 892 samples) and TDI sum score $(p=0.001$, based on 892 samples) ipsilaterally with the side of the choanal atresia. There was no significant difference in terms of odor discrimination, identification, or olfactory bulb volume. Olfactory performance and the volume of the olfactory
90

ORL 2022;84:89-92

DOI: $10.1159 / 000520188$
Georgiopoulos/Postler/Rombaux/ Gudziol/Abolmaali/Hummel 
bulbs are summarized in Table 1. ROI analysis of VBM data showed that the hippocampus was significantly smaller ( $p=0.001$, based on 884 samples) ipsilaterally with the side of the choanal atresia (mean volume on the healthy side: $3.34 \pm 0.39 \mathrm{~cm}^{3}$; mean volume on the affected side: $3.01 \pm 0.3 \mathrm{~cm}^{3}$ ). There were no differences in grey matter volume within the entorhinal cortex and amygdala. The ROI volumes for each participant are also presented in Table 1.

\section{Discussion}

In this pilot study, we present 3 cases of unilateral choanal atresia with significantly lower odor threshold postsurgically. To date, there are only 2 published studies focusing on the postsurgical olfactory performance of adults with choanal atresia $[11,12]$. Both of them have reported that patients with unilateral atresia have normal olfactory performance. However, both studies have included a very small number of patients with unilateral atresia ( 1 patient and 2 patients, respectively). Moreover, olfactory evaluation has not been extensive in any of these studies. Gross-Isseroff et al. [11] have tested their subject only in terms of odor threshold separately for each nostril, while Leclerc et al. [12] have tested their 2 subjects with an odor identification test (both nostrils at once). Based on our findings, we suggest that olfactory testing of these patients should focus on all aspects of olfaction (identification, threshold, and discrimination) and should be performed separately for each nostril.

The olfactory system is highly plastic. The volume of the olfactory bulb changes accordingly with the sensory input (or the lack of it thereby) $[8,16,17]$. Similarly, the volume of olfactory related brain areas is typically low when olfaction is impaired, for instance in neurodegenerative disorders [18]. Brain volumetric analysis of the present cohort revealed grey matter reductions within the hippocampus, on the same side with the choanal atresia. Given the small sample, it is possible that this finding is incidental. Future studies with more patients are needed in order to further investigate potential structural brain abnormalities associated with choanal atresia.

Our results raise the question of the possible pathophysiology behind the observed lower hippocampal volume on the affected side. According to a previous study by Han et al. [19] on patients with subjective hyperosmia, the processing of olfactory input can affect the size of the hippocampus. Furthermore, unlike other sensory sys- tems, the olfactory cortex maintains its connectivity to the hippocampus, and this connectivity varies with the respiratory phase [20]. Given the lack of other comorbidity that could explain hippocampal volume loss in our cohort, one could assume that lower hippocampal volumes ipsilaterally with the side of choanal atresia could be explained by reduced sensory input or by the lack of nasal breathing throughout childhood, or perhaps by their combination. However, the observed lower odor threshold and TDI scores on the affected side advocate a potential connection between lower sensory input and lower hippocampal volume.

Interestingly, all 3 patients could experience odors in a typical manner, being able to identify most odors even at the side of the atresia. This may indicate that aspects of odor perception might to some extent be independent of exposure during early childhood/adolescence. In contrast, odor threshold was significantly lower on the side of the atresia. As threshold measurements appear to best reflect the function of the peripheral olfactory system [21], they may potentially be more sensitive in depicting olfactory deficits in these patients.

Another interesting observation is that patient 3, who underwent surgery at the age of 9 years, performed better both in terms of odor threshold and odor discrimination compared to the other 2 participants who were operated in adulthood. This finding further highlights the plasticity of the olfactory system. Also, it could serve as an argument to recommend early surgery in unilateral choanal atresia in order to preserve olfactory function.

An obvious source of weakness in this pilot study is the small number of participants. Choanal atresia is a rare malformation, which is a limiting factor for large observational studies. Despite that, our cohort is actually larger than the 2 previous studies in this field. Another limitation of the small sample size extends to the value of the application of any statistical tools for behavioral data analysis. Moreover, this study does not include longitudinal measurements or data on other postsurgical effects (e.g., nasal flow). However, despite the exploratory nature that this study has, we believe that our findings provide new, useful insight regarding olfactory function after surgical intervention.

In conclusion, this pilot study provides evidence of potentially subtle, yet detectable, long-term olfactory deficits in patients with unilateral choanal atresia, several years after surgical intervention. More studies with larger populations are needed in order to shed light in this understudied field. 


\section{Statement of Ethics}

The study was conducted in accordance with the World Medical Association Declaration of Helsinki and was approved by the Ethics Committee at the University Clinic of the TU Dresden (Application No. EK 96032015). All participants gave their written informed consent.

\section{Conflict of Interest Statement}

The authors have no conflicts of interest to declare.

\section{Funding Sources} dation.

This study was funded by the Alexander von Humboldt Foun-

\section{References}

1 Harris J, Robert E, Källén B. Epidemiology of choanal atresia with special reference to the CHARGE association. Pediatrics. 1997 Mar; 99(3):363-7.

2 Case AP, Mitchell LE. Prevalence and patterns of choanal atresia and choanal stenosis among pregnancies in Texas, 1999-2004. Am I Med Genet A. 2011 Apr;155A(4):786-91.

3 Ramsden JD, Campisi P, Forte V. Choanal atresia and choanal stenosis. Otolaryngol Clin North Am. 2009 Apr;42(2):339-52, x.

4 Keller JL, Kacker A. Choanal atresia, CHARGE association, and congenital nasal stenosis. Otolaryngol Clin North Am. 2000 Dec;33(6):1343-51, viii.

5 Kurosaka H. Choanal atresia and stenosis: development and diseases of the nasal cavity. Wiley Interdiscip Rev Dev Biol. 2019 Jan;8(1): e336.

6 Anajar S, Hassnaoui J, Rouadi S, Abada R, Roubal M, Mahtar M. A rare case report of bilateral choanal atresia in an adult. Int J Surg Case Rep. 2017;37:127-9.

7 von Gudden JBA. Experimentaluntersuchungen über das peripherische und centrale Nervensystem. Arch Psychiatr Nervenkr. 1870 Feb 3;2:693-723.

8 Huart C, Rombaux P, Hummel T. Plasticity of the human olfactory system: the olfactory bulb. Molecules. 2013 Sep 17;18(9):11586600 .

\section{Author Contributions}

C.G. contributed to data analysis and interpretation, as well as drafting and revising the article. M.P., V.G., and N.A. contributed to data collection and interpretation, as well as critical revision of the article. P.R. contributed to study conception and critical revision of the article. T.H. contributed to study conception, data collection, and interpretation, as well as critical revision of the article. All authors have approved the final version of this article.

\section{Data Availability Statement}

Demographic and behavioral data are reported in the article. MRI data are not publicly available on legal grounds.
9 Chalouhi C, Faulcon P, Le Bihan C, HertzPannier L, Bonfils P, Abadie V. Olfactory evaluation in children: application to the CHARGE syndrome. Pediatrics. 2005 Jul; 116(1):e81-8.

10 Pinto G, Abadie V, Mesnage R, Blustajn J, Cabrol S, Amiel J, et al. CHARGE syndrome includes hypogonadotropic hypogonadism and abnormal olfactory bulb development. J Clin Endocrinol Metab. 2005 Oct;90(10):5621-6.

11 Gross-Isseroff R, Ophir D, Marshak G, Ganchrow JR, Beizer M, Lancet D. Olfactory function following late repair of choanal atresia. Laryngoscope. 1989 Nov;99(11):1165-6.

12 Leclerc JE, Leclerc JT, Bernier K. Choanal atresia: long-term follow-up with objective evaluation of nasal airway and olfaction. Otolaryngol Head Neck Surg. 2008 Jan;138(1): 43-9.

13 Burrow TA, Saal HM, de Alarcon A, Martin LJ, Cotton RT, Hopkin RJ. Characterization of congenital anomalies in individuals with choanal atresia. Arch Otolaryngol Head Neck Surg. 2009 Jun;135(6):543-7.

14 Oleszkiewicz A, Schriever VA, Croy I, Hähner A, Hummel T. Updated Sniffin' Sticks normative data based on an extended sample of 9139 subjects. Eur Arch Otorhinolaryngol. 2019 Mar;276(3):719-28.
15 Rombaux P, Duprez T, Hummel T. Olfactory bulb volume in the clinical assessment of olfactory dysfunction. Rhinology. 2009 Mar; 47(1):3-9.

16 Negoias S, Pietsch K, Hummel T. Changes in olfactory bulb volume following lateralized olfactory training. Brain Imaging Behav. 2017 Aug;11(4):998-1005.

17 Fitzwater E, Coppola DM. Olfactory deprivation and enrichment: an identity of opposites? Chem Senses. 2020 Oct 26;46:71.

18 Lee EY, Eslinger PJ, Du G, Kong L, Lewis MM, Huang X. Olfactory-related cortical atrophy is associated with olfactory dysfunction in Parkinson's disease. Mov Disord. 2014 Aug; 29(9):1205-8.

19 Han P, Stiller-Stut FP, Fjaeldstad A, Hummel T. Greater hippocampal gray matter volume in subjective hyperosmia: a voxel-based morphometry study. Sci Rep. 2020 Nov 2;10(1): 18869.

20 Zhou GY, Olofsson JK, Koubeissi MZ, Menelaou G, Rosenow J, Schuele SU, et al. Human hippocampal connectivity is stronger in olfaction than other sensory systems. Prog Neurobiol. 2021 Jun;201:102027.

21 Moberg PJ, Agrin R, Gur RE, Gur RC, Turetsky BI, Doty RL. Olfactory dysfunction in schizophrenia: a qualitative and quantitative review. Neuropsychopharmacology. 1999 Sep;21(3):325-40. 